\title{
THE CONFIGURATION SPACE OF EQUILATERAL AND EQUIANGULAR OCTAGONS
}

\section{Yasuhiko Kamiyama}

Department of Mathematics

University of the Ryukyus

Nishihara-Cho, Okinawa 903-0213

Japan

e-mail:kamiyama@sci.u-ryukyu.ac.jp

\begin{abstract}
In [4], we determined the topological type of the configuration space of equilateral and equiangular heptagons for generic bond angle. In this paper, we determine the topological type of the configuration space of equilateral and equiangular octagons for the case when the bond angle $\theta$ satisfies $\frac{\pi}{2}<\theta<\frac{3}{4} \pi$.
\end{abstract}

\section{Introduction}

In [4], we determined the topological type of the configuration space of equilateral and equiangular heptagons for generic bond angle. We also studied its related space. Since these spaces have dimension one, they are disjoint union of a certain number of $S^{1}$ s. Hence the problem is to determine the number of connected components.

Received: August 6, 2021; Accepted: September 8, 2021

2020 Mathematics Subject Classification: Primary 58D29; Secondary 55R80.

Keywords and phrases: polygon space, bond angle, topological type.

Published Online: September 10, 2021 
In this paper, we determine the topological type of the configuration space of equilateral and equiangular octagons for the case that the bond angle is slightly smaller than $\frac{3}{4} \pi$. We also study its related space. Since these spaces have dimension two, the problem is not only the number of connected components but also the genus of a connected component.

Configuration spaces of mechanical linkages in the Euclidean space of dimension three, also known as polygon spaces, are quite important in various engineering applications: in molecular biology, they describe varieties of molecular shapes, in robotics, they appear as spaces of all possible configurations of some mechanisms, and they play a central role in statistical shape theory.

Mathematically, these spaces are also very interesting: The symplectic structure on polygon spaces was studied in the seminal paper [6]. We refer to [2] for an excellent exposition with emphasis on Morse theory.

Recently, in order to construct a mathematical model of chemical objects, attempts are made to impose restrictions on the bond angles of a polygon. In this case, we usually assume that a polygon is equilateral.

Generalizing the configuration space which was already constructed, we give the following filtration of the usual polygon space: For $\theta \in(0, \pi)$ and $s \in\{0,1, \ldots, n\}$, we set

$A_{n}^{S}(\theta):=\left\{\left(u_{1}, \ldots, u_{n}\right) \in\left(S^{2}\right)^{n} \mid\right.$ the following (i) and (ii) hold $\}$.

(i) $\sum_{i=1}^{n} u_{i}=0$.

(ii) $\left\langle u_{i}, u_{i+1}\right\rangle=-\cos \theta$ for $1 \leq i \leq s$.

Here $\langle$,$\rangle denotes the standard inner product on \mathbb{R}^{3}$. Moreover, when $s=n$, we understand $\left\langle u_{n}, u_{n+1}\right\rangle$ to be $\left\langle u_{n}, u_{1}\right\rangle$. 
The Configuration Space of Equilateral and Equiangular Octagons 119

Let $S O(3)$ act on $A_{n}^{S}(\theta)$ diagonally and we set

$$
\mathcal{P}_{n}^{S}(\theta):=A_{n}^{S}(\theta) / S O(3)
$$

Note that $\mathcal{P}_{n}^{0}(\theta)$ is the usual polygon space.

We consider the case that $s$ is an element of $\{n-1, n\}$. First, the following result is known:

Theorem 1 ([3, Theorems A and B]). Let $s$ be an element of $\{n-1, n\}$. Then the following assertions hold:

(i) Let $n$ be an odd integer greater than or equal to 7. Then $\mathcal{P}_{n}^{S}(\theta) \neq \varnothing$ if and only if $\theta \in\left[\frac{\pi}{n}, \frac{n-2}{n} \pi\right]$.

(ii) Let $n$ be an even integer greater than or equal to 4. Then $\mathcal{P}_{n}^{s}(\theta) \neq \varnothing$ if and only if $\theta \in\left(0, \frac{n-2}{n} \pi\right]$.

Second, the following results are known:

(i) (a) The topological type of $\mathcal{P}_{n}^{n}(\theta)$ for all $\theta$ was determined by Crippen [1] for $n \leq 5$ and by O'Hara [7] for $n=6$.

(b) Crippen also determined the topological type of $\mathcal{P}_{7}^{7}(\theta)$ when $\theta$ is the ideal tetrahedral bond angle $\arccos \left(-\frac{1}{3}\right) \approx 109.47^{\circ}$. Generalizing the result, [4] determined the topological type of $\mathcal{P}_{7}^{7}(\theta)$ for generic $\theta$. The result is given as follows: There is a homeomorphism

$$
\mathcal{P}_{7}^{7}(\theta) \cong \begin{cases}2 S^{1}, & \text { if } \frac{3}{7} \pi<\theta<\frac{5}{7} \pi, \\ 8 S^{1}, & \text { if } \frac{\pi}{3}<\theta<\frac{3}{7} \pi, \\ 2 S^{1}, & \text { if } \frac{\pi}{7}<\theta<\frac{\pi}{3} .\end{cases}
$$


Here throughout this paper, we use the following notations: For spaces $A_{1}$ and $A_{2}$, the notation $A_{1} \cong A_{2}$ means that $A_{1}$ is homeomorphic to $A_{2}$. Moreover, if $k$ is an integer which is greater than 1 , the notation $k A$ means the disjoint union of $k$ copies of $A$.

(ii) Until [4], very little was known about the topology of $\mathcal{P}_{n}^{n-1}(\theta)$. In [4], we determined the topological type of $\mathcal{P}_{6}^{5}(\theta)$ for generic $\theta$. The result is given as follows: There is a homeomorphism

$$
\mathcal{P}_{6}^{5}(\theta) \cong \begin{cases}2 S^{1}, & \text { if } \frac{\pi}{3}<\theta<\frac{2}{3} \pi, \\ 4 S^{1}, & \text { if } 0<\theta<\frac{\pi}{3} .\end{cases}
$$

See [4] for the results on the topological type of $\mathcal{P}_{n}^{S}(\theta)$ for $s \leq n-2$.

The purpose of this paper is to generalize (1) to the case of $\mathcal{P}_{8}^{8}(\theta)$ when

$\theta$ is near $\frac{3}{4} \pi$. We also generalize (2) to the topological type of $\mathcal{P}_{7}^{6}(\theta)$ for generic $\theta$. Note that these spaces have dimension two.

This paper is organized as follows. In Section 2, we state our main results, and in Section 3, we prove them.

\section{Main Results}

In what follows, let $(a, b)$ denote the open interval.

Theorem A. Assume that the bond angle $\theta$ satisfies $\frac{\pi}{2}<\theta<\frac{3}{4} \pi$. Then we have the following homeomorphism:

$$
\mathcal{P}_{8}^{8}(\theta) \cong S^{2} .
$$

In particular,

$$
\mathcal{P}_{8}^{8}\left(\arccos \left(-\frac{1}{3}\right)\right) \cong S^{2}
$$


The Configuration Space of Equilateral and Equiangular Octagons 121

Theorem B. The topological type of $\mathcal{P}_{7}^{6}(\theta)$ for generic $\theta$ is given by the following Table 1.

Table 1. The topological type of $\mathcal{P}_{7}^{6}(\theta)$

\begin{tabular}{|c|c|c|c|c|c|}
\hline Range of $\theta$ & $\left(\frac{\pi}{7}, 0.7227\right)$ & $\left(0.7227, \frac{\pi}{3}\right)$ & $\left(\frac{\pi}{3}, \frac{3}{7} \pi\right)$ & $\left(\frac{3}{7} \pi, 1.779\right)$ & $\left(1.779, \frac{5}{7} \pi\right)$ \\
\hline Top. type & $\Sigma_{1}$ & $\Sigma_{9}$ & $\Sigma_{9}$ & $\Sigma_{5}$ & $\Sigma_{1}$ \\
\hline
\end{tabular}

Here $\Sigma_{g}$ denotes the closed connected orientable surface of genus $g$.

Remark 2. (i) From Theorems A and B, the spaces in these theorems are in fact connected.

(ii) About Theorem A, we shall prove in a subsequent paper [5] that $\mathcal{P}_{8}^{8}(\theta)$ has a singular point if $\theta$ satisfies $0<\theta \leq \frac{\pi}{2}$.

(iii) Theorem B tells us that $\mathcal{P}_{7}^{6}\left(\frac{\pi}{2}\right)$ is a manifold, but $\mathcal{P}_{7}^{6}\left(\frac{\pi}{3}\right)$ has a singular point.

Before we leave this section, we outline the proofs of Theorems A and B for $1.779<\theta<\frac{5}{7} \pi$. In Section 3, we construct the functions

$$
\mu: \mathcal{P}_{8}^{8}(\theta) \rightarrow \mathbb{R} \quad \text { and } \quad v: \mathcal{P}_{7}^{6}(\theta) \rightarrow \mathbb{R}
$$

and study the fibers $\mu^{-1}(r)$ and $v^{-1}(r)$ for each $r \in \mathbb{R}$. In order to state the result, we define $X$ to be the space given by the following Figure 1 .

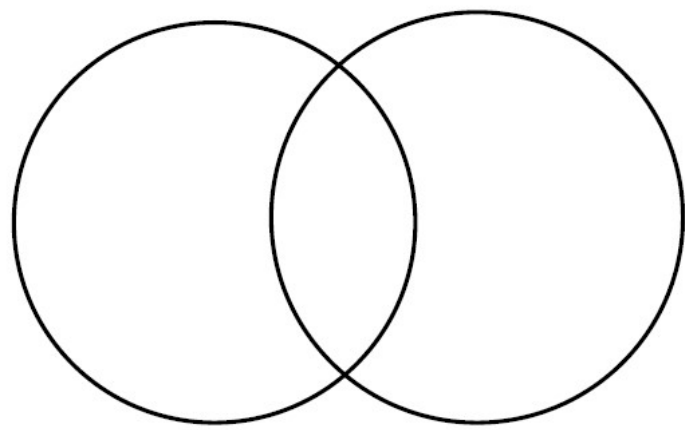

Figure 1. The space $X$. 
If $\frac{\pi}{2}<\theta<\frac{3}{4} \pi$, then there exists a positive real number $a$ such that the following (4) holds. (See also Table 2 in Section 3.)

$$
\mu^{-1}(r) \cong \begin{cases}\varnothing, & \text { if }|r|>a, \\ \{\text { two points }\}, & \text { if }|r|=a, \\ 2 S^{1}, & \text { if } 0<|r|<a, \\ X, & \text { if } r=0 .\end{cases}
$$

Moreover, as shown in the left figure of Figure 2, the self-intersection points of $X$ are saddle points in $\mathcal{P}_{8}^{8}(\theta)$. Hence, we obtain Theorem A.

On the other hand, if $1.779<\theta<\frac{5}{7} \pi$, then there exists a positive real number $b$ such that the following (5) holds. (See also Table 3 in Section 3.)

$$
v^{-1}(r) \cong \begin{cases}\varnothing, & \text { if }|r|>b, \\ \{\text { one point }\}, & \text { if }|r|=b, \\ S^{1}, & \text { if } 0<|r|<b, \\ X, & \text { if } r=0 .\end{cases}
$$

Moreover, as shown in the right figure of Figure 2, the self-intersection points of $X$ are also saddle points in $\mathcal{P}_{7}^{6}(\theta)$. Hence, we obtain Theorem B for $1.779<\theta<\frac{5}{7} \pi$.
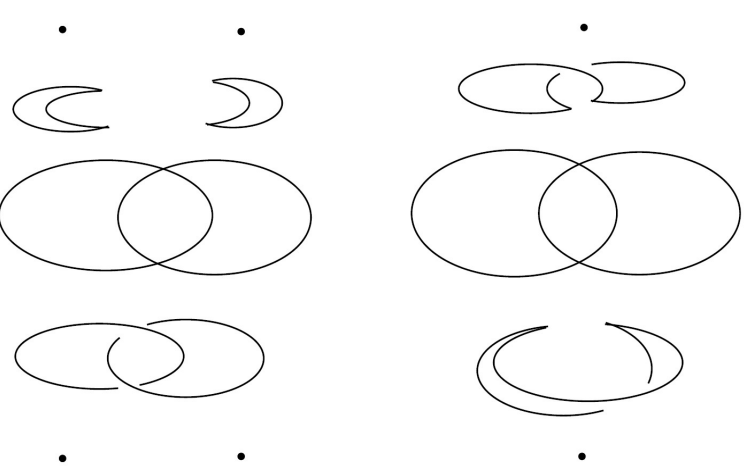

Figure 2. $\mathcal{P}_{8}^{8}(\theta)$ (left figure) and $\mathcal{P}_{7}^{6}(\theta)$ (right figure). 


\section{Proofs of the Main Results}

First, we explain that for two reasons, Morse theory is not enough in order to prove Theorems A and B. The first reason is that it is not clear that $\mathcal{P}_{n}^{S}(\theta)$ for generic $\theta$ is a manifold because it is not easy to prove the fact that polynomial equations for $\mathcal{P}_{n}^{S}(\theta)$ intersect transversely.

The second reason is that since a generic function on a manifold is a Morse function, we can calculate $\chi\left(\mathcal{P}_{n}^{S}(\theta)\right)$ for $(n, s)=(8,8)$ or $(7,6)$. But it is not easy to prove that these spaces are connected.

In order to avoid these difficulties, we construct the function

$$
f: \mathcal{P}_{n}^{s}(\theta) \rightarrow S^{1}
$$

and study the fiber $f^{-1}(\zeta)$ for each $\zeta \in S^{1}$. We set

$$
\mathcal{Q}_{n}^{s}(\theta):=\left\{\left(u_{1}, \ldots, u_{n}\right) \in A_{n}^{S}(\theta) \mid u_{4}=(-\cos \theta,-\sin \theta, 0) \text { and } u_{5}=(1,0,0)\right\}
$$

By the $S O(3)$-action on $A_{n}^{S}(\theta)$, we may normalize $u_{4}$ and $u_{5}$ as in (6). Hence, we have

$$
\mathcal{P}_{n}^{S}(\theta) \cong \mathcal{Q}_{n}^{S}(\theta)
$$

Hereafter we consider $\mathcal{Q}_{n}^{S}(\theta)$ for $\mathcal{P}_{n}^{S}(\theta)$.

For $u_{4}$ in (6), we set

$$
L(\theta):=\left\{P \in S^{2} \mid\left\langle P, u_{4}\right\rangle=-\cos \theta\right\}
$$

It is clear that $L(\theta) \cong S^{1}$. We specify the parametrization of $L(\theta)$ as follows: We set

$$
c(\varphi):=\left(\cos ^{2} \theta-\sin ^{2} \theta \cos \varphi, \cos \theta \sin \theta(1+\cos \varphi), \sin \theta \sin \varphi\right) .
$$

Then $L(\theta)$ is parametrized by $c:[-\pi, \pi] \rightarrow L(\theta)$. 
We define the function $f: \mathcal{Q}_{n}^{s}(\theta) \rightarrow L(\theta)$ by

$$
f\left(u_{1}, \ldots, u_{n}\right)=u_{3} \text {. }
$$

Using this, we set

$$
M_{n, s, \theta}(\varphi):=f^{-1}(c(\varphi)) .
$$

We claim that the following homeomorphism holds:

$$
M_{n, s, \theta}(\varphi) \cong M_{n, s, \theta}(-\varphi) \text { for all } \varphi \in[-\pi, \pi] .
$$

In fact, there is an involution

$$
\tau: \mathcal{Q}_{n}^{S}(\theta) \rightarrow \mathcal{Q}_{n}^{S}(\theta)
$$

defined by

$$
\tau\left(u_{1}, u_{2}, \ldots, u_{n}\right)=\left(\bar{u}_{1}, \bar{u}_{2}, \ldots, \bar{u}_{n}\right) .
$$

Here for $P=(x, y, z) \in S^{2}$, we set $\bar{P}:=(x, y,-z)$. Since $c(-\varphi)=$ $\overline{c(\varphi)}$, the restriction of $\tau$ gives a homeomorphism

$$
\left.\tau\right|_{M_{n, s, \theta}(\varphi)}: M_{n, s, \theta}(\varphi) \rightarrow M_{n, s, \theta}(-\varphi)
$$

Hence (7) holds.

Proof of Theorem A. Assume that the bond angle $\theta$ satisfies $\frac{\pi}{2}<\theta$ $<\frac{3}{4} \pi$. Then with the aid of a computer, we can prove the following Table 2 .

Table 2. The topological type of $M_{8,8, \theta}(\varphi)$

\begin{tabular}{|c|c|}
\hline Range of $\varphi$ & Top. type of $M_{8,8, \theta}(\varphi)$ \\
\hline$\varphi_{0}<|\varphi| \leq \pi$ & $\varnothing$ \\
\hline$\pm \varphi_{0}$ & \{two points\} \\
\hline $0<|\varphi|<\varphi_{0}$ & $2 S^{1}$ \\
\hline 0 & $X$ \\
\hline
\end{tabular}


The Configuration Space of Equilateral and Equiangular Octagons 125

Here the space $X$ is defined by Figure 1. Moreover, with further use of a computer, we can check that the self-intersection points of $X$ are saddle points in $\mathcal{Q}_{8}^{8}(\theta)$. (See the left figure of Figure 2.) Hence, we have $\mathcal{Q}_{8}^{8}(\theta) \cong S^{2}$.

Remark 3. We have two remarks about Table 2. First, the table indeed satisfies (7). Second, the table shows that for $f: \mathcal{Q}_{8}^{8}(\theta) \rightarrow L(\theta)$, we have $c(\pi) \notin f\left(\mathcal{Q}_{8}^{8}(\theta)\right)$. Then we define the function $\mu$ in (3) by $\mu:=\left(\left.c\right|_{(-\pi, \pi)}\right)^{-1} \circ f$.

Proof of Theorem B. (I) The case that $1.779<\theta<\frac{5}{7} \pi$.

There exists $\varphi_{0} \in(0, \pi)$ such that the following Table 3 holds.

Table 3. The topological type of $M_{7,6, \theta}(\varphi)$ for $1.779<\theta<\frac{5}{7} \pi$

\begin{tabular}{|c|c|}
\hline Range of $\varphi$ & Top. type of $M_{7,6, \theta}(\varphi)$ \\
\hline$\varphi_{0}<|\varphi| \leq \pi$ & $\varnothing$ \\
\hline$\pm \varphi_{0}$ & \{one point \\
\hline $0<|\varphi|<\varphi_{0}$ & $S^{1}$ \\
\hline 0 & $X$ \\
\hline
\end{tabular}

Similar to the proof of Theorem A, we can check that the selfintersection points of $X$ are also saddle points in $\mathcal{Q}_{7}^{6}(\theta)$. (See the right figure of Figure 2.) Hence, we have $\mathcal{Q}_{7}^{6}(\theta) \cong \Sigma_{1}$.

(II) The case that $\frac{\pi}{2}<\theta<1.779$.

There exist $\varphi_{0}, \varphi_{1}$ and $\varphi_{2}$ (where $\left.0<\varphi_{0}<\varphi_{1}<\varphi_{2}<\pi\right)$ such that the following Table 4 holds. 
Table 4. The topological type of $M_{7,6, \theta}(\varphi)$ for $\frac{\pi}{2}<\theta<1.779$

\begin{tabular}{|c|c|}
\hline Range of $\varphi$ & Top. type of $M_{7,6, \theta}(\varphi)$ \\
\hline$\varphi_{2}<|\varphi| \leq \pi$ & $\varnothing$ \\
\hline$\pm \varphi_{2}$ & \{one point \\
\hline$\varphi_{1}<|\varphi|<\varphi_{2}$ & $S^{1}$ \\
\hline$\pm \varphi_{1}$ & $Y$ \\
\hline$\varphi_{0}<|\varphi|<\varphi_{1}$ & $3 S^{1}$ \\
\hline$\pm \varphi_{0}$ & $2 S^{1} \amalg\{$ one point \\
\hline $0<|\varphi|<\varphi_{0}$ & $2 S^{1}$ \\
\hline 0 & $2 X$ \\
\hline
\end{tabular}

Here we define $Y$ to be the space given by the following Figure 3 .

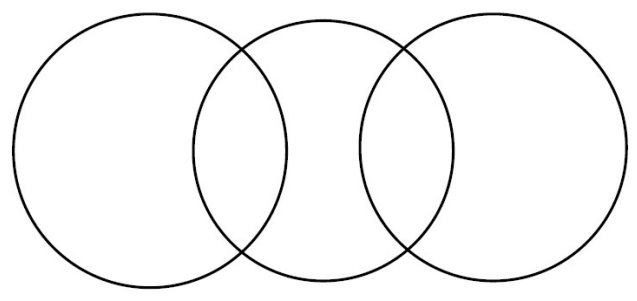

Figure 3. The space $Y$.

Applying the Mayer-Vietoris argument to Table 4, we have $\chi\left(\mathcal{Q}_{7}^{6}(\theta)\right)$ $=-8$. The table also tells us that $\mathcal{Q}_{7}^{6}(\theta)$ is connected. Hence, we have $\mathcal{Q}_{7}^{6}(\theta) \cong \Sigma_{5}$.

(III) The case that $\theta=\frac{\pi}{2}$.

In Table 4 , we set $\varphi_{2}=\pi$ and understand the part $M_{7,6, \theta}\left(\varphi_{2}\right)=$ \{one point $\}$ to be $M_{7,6, \theta}(\pi)=$ \{two points\}. Then we obtain the table for the case $\theta=\frac{\pi}{2}$. From this, we have $\mathcal{Q}_{7}^{6}\left(\frac{\pi}{2}\right) \cong \Sigma_{5}$. 
(IV) The case that $\frac{3}{7} \pi<\theta<\frac{\pi}{2}$.

There exist $\varphi_{0}, \varphi_{1}$ and $\varphi_{2}$ (where $0<\varphi_{0}<\varphi_{1}<\varphi_{2}<\pi$ ) such that the following Table 5 holds.

Table 5. The topological type of $M_{7,6, \theta}(\varphi)$ for $\frac{3}{7} \pi<\theta<\frac{\pi}{2}$

\begin{tabular}{|c|c|}
\hline Range of $\varphi$ & Top. type of $M_{7,6, \theta}(\varphi)$ \\
\hline$\pi$ & $2 S^{1}$ \\
\hline$\varphi_{2}<|\varphi|<\pi$ & $2 S^{1}$ \\
\hline$\pm \varphi_{2}$ & $S^{1} \amalg\{$ one point $\}$ \\
\hline$\varphi_{1}<|\varphi|<\varphi_{2}$ & $S^{1}$ \\
\hline$\pm \varphi_{1}$ & $Y$ \\
\hline$\varphi_{0}<|\varphi|<\varphi_{1}$ & $3 S^{1}$ \\
\hline$\pm \varphi_{0}$ & $2 S^{1} \amalg\{$ one point $\}$ \\
\hline $0<|\varphi|<\varphi_{0}$ & $2 S^{1}$ \\
\hline 0 & $X$ \\
\hline
\end{tabular}

Moreover, the union of $M_{7,6, \theta}(\varphi)$ for $\varphi_{1}<|\varphi| \leq \pi$ is given by the following Figure 4 . Combining Table 5 and the figure, we have $\mathcal{Q}_{7}^{6}(\theta) \cong \Sigma_{5}$.
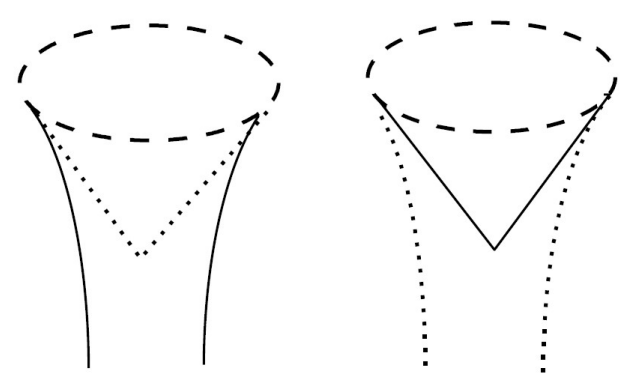

Figure 4. The union of $M_{7,6, \theta}(\varphi)$ for $\varphi_{1}<|\varphi| \leq \pi$. The broken line, solid line and dotted line illustrate $M_{7,6, \theta}(\varphi)$ for $\varphi=\pi, \varphi_{1}<\varphi<\pi$ and $-\pi<\varphi<-\varphi_{1}$, respectively. 
Remark 4. We study how the neighborhood of $M_{7,6, \theta}(\pi)$ changes as $\theta$ decreases from (IV) to (II). As $\theta$ decreases, the broken lines and cuffs in Figure 4 shrink. When $\theta=\frac{\pi}{2}$, they degenerate to the two points in (III). If $\theta$ decreases further, then the height $\varphi$ of the points gets lower.

(V) The case that $\frac{\pi}{3}<\theta<\frac{3}{7} \pi$.

There exist $\varphi_{0}, \varphi_{1}$ and $\varphi_{2}$ (where $\left.0<\varphi_{0}<\varphi_{1}<\varphi_{2}<\pi\right)$ such that the following Table 6 holds:

Table 6. The topological type of $M_{7,6, \theta}(\varphi)$ for $\frac{\pi}{3}<\theta<\frac{3}{7} \pi$

\begin{tabular}{|c|c|}
\hline Range of $\varphi$ & Top. type of $M_{7,6, \theta}(\varphi)$ \\
\hline$\pi$ & $2 S^{1}$ \\
\hline$\varphi_{2}<|\varphi|<\pi$ & $2 S^{1}$ \\
\hline$\pm \varphi_{2}$ & $S^{1} \amalg Y$ \\
\hline$\varphi_{1}<|\varphi|<\varphi_{2}$ & $4 S^{1}$ \\
\hline$\pm \varphi_{1}$ & $3 S^{1} \amalg\{$ one point $\}$ \\
\hline$\varphi_{0}<|\varphi|<\varphi_{1}$ & $3 S^{1}$ \\
\hline$\pm \varphi_{0}$ & $Y$ \\
\hline $0<|\varphi|<\varphi_{0}$ & $S^{1}$ \\
\hline 0 & $X$ \\
\hline
\end{tabular}

Applying the Mayer-Vietoris argument to Table 6, we have $\chi\left(\mathcal{Q}_{7}^{6}(\theta)\right)$ $=-16$. By the same argument as in Figure 4, we have $\mathcal{Q}_{7}^{6}(\theta) \cong \Sigma_{9}$.

(VI) The case that $0.7227<\theta<\frac{\pi}{3}$.

The table for the case is same as Table 6 . 
(VII) The case that $\frac{\pi}{7}<\theta<0.7227$.

The table for the case is same as Table 3 .

Remark 5. Consider $\mathcal{Q}_{n}^{s}(\theta)$ for $(n, s)=(7,7)$ or $(6,5)$. In this case, it is proved in [4] that for generic $\theta$, we have

$$
\mathcal{Q}_{n}^{s}(\theta) \cong \frac{\left|M_{n, s, \theta}(0)\right|+\left|M_{n, s, \theta}(\pi)\right|}{2} S^{1},
$$

where $|-|$ stands for the cardinality. (8) implies the following result: For such $(n, s)$, we have similar tables as Tables 2-6, which depend on certain $\varphi_{i}$ 's and the level set $M_{n, s, \theta}(\varphi)$ consists of a certain number of points. But in spite of the $\varphi_{i}$ 's, the topological type of $\mathcal{Q}_{n}^{S}(\theta)$ depends only on $M_{n, s, \theta}(0)$ and $M_{n, s, \theta}(\pi)$.

\section{References}

[1] G. M. Crippen, Exploring the conformation space of cycloalkanes by linearized embedding, J. Comput. Chem. 13 (1992), 351-361.

[2] M. Farber, Invitation to topological robotics, Zurich Lectures in Advanced Mathematics, European Mathematical Society (EMS), 2008.

[3] Y. Kamiyama, A filtration of the configuration space of spatial polygons, Adv. Appl. Discrete Math. 22(1) (2019), 67-74.

[4] Y. Kamiyama, The configuration space of equilateral and equiangular heptagons, JP J. Geom. Topol. 25(1-2) (2020), 25-33.

[5] Y. Kamiyama, On the singularity of the configuration space of equilateral and equiangular polygons, Advances and Applications in Discrete Mathematics (submitted).

[6] M. Kapovich and J. Millson, The symplectic geometry of polygons in the Euclidean space, J. Differential Geom. 44 (1996), 479-513.

[7] J. O'Hara, The configuration space of equilateral and equiangular hexagons, Osaka J. Math. 50 (2013), 477-489. 